Review

\title{
Long-Term Storage Considerations for Spacecraft Lubricants
}

\author{
Michael Buttery *, Simon Lewis, Anthony Kent, Rachel Bingley and Matthew Cropper \\ European Space Tribology Laboratory (ESTL), ESR Technology, 202 Cavendish Place, Birchwood Park, \\ Warrington WA3 6WU, UK; simon.lewis@esrtechnology.com (S.L.); anthony.kent@esrtechnology.com (A.K.); \\ rachel.bingley@esrtechnology.com (R.B.); matthew.cropper@esrtechnology.com (M.C.) \\ * Correspondence: Michael.buttery@esrtechnology.com; Tel.: +44-(0)-1925-84-3437
}

Received: 28 February 2020; Accepted: 13 March 2020; Published: 13 March 2020

\begin{abstract}
Spacecraft mechanisms commonly undergo extended periods of storage, either on-ground, or in-flight and there are an increasing number of missions for which some element of long-term storage may be required. Despite the obvious potential for degradation of lubricants during storage which might impact mechanism functionality or life and so even become mission-threatening, today's understanding of storage phenomena is rather incomplete. This paper provides consolidation and review of recent experimental studies in this area and considers the range of storage conditions and associated degradation phenomena which could impact different lubricants. Whilst some storage best practice guidelines exist, experimental verification of the impact of storage phenomena has rarely been carried out and test data is rather scarce and incomplete. Given the absence of comprehensive data to support design, lubricant selection or the development of storage protocols, it is shown that for all lubricant types careful control of storage and test environments combined with monitoring of the evolving tribological performance during periodic mechanism exercising are presently the most effective storage risk mitigations.
\end{abstract}

Keywords: lubrication; spacecraft; vacuum; tribology; long-term storage

\section{Introduction}

Spacecraft mechanism components are commonly stored before or after spacecraft integration for a considerable time. Such storage can occur prior to launch (on-ground storage), or in-flight where a mechanism remains dormant but exposed to the flight environment potentially for some years prior to operation. Whilst the "ideally benign" on-ground storage conditions for the most sensitive lubricants (e.g., sputtered $\mathrm{MoS}_{2}$ ) are typically considered as dry nitrogen $\left(\mathrm{DN}_{2}\right)$ of $1-3 \%$ ppm moisture, for practical reasons some relaxation of this criteria is commonly followed. Extended on-ground storage at spacecraft level is often under a continuous long-term purge of semi-dry nitrogen ( 10-20\% relative humidity $(\mathrm{RH})$ ), with periodic "exercising" of the components under $\mathrm{DN}_{2}$. For in-flight storage, mechanisms are exposed to the thermal vacuum conditions of space, but usually without any possibility for "exercise". Storage periods can continue for months and occasionally for many years both before and after launch.

The general consideration is that this storage period under "benign" conditions will have a negligible impact on the tribological behaviour of the employed lubricants (assuming they are correctly applied). However recent activities (at ESTL and elsewhere) have cast some doubt onto this understanding, for both storage and operational environment conditions. There are growing concerns over the impact of on-ground or in-flight storage on subsequent performance, especially where such storage is for longer than the originally specified design requirement for the mechanism and/or under sub-optimal conditions. 
The areas of concern regarding the change in the tribological performance of lubricants can be grouped into the following three categories.

- Operational Environment-the environment in which the lubricant is sheared during operation (e.g., $\mathrm{DN}_{2}$ for ground testing, vacuum for flight).

- On-ground Storage Environment-non-operational exposure environment prior to shearing. Lubricants may be stored either fully applied to the respective components (such as for angular contact bearings), or prior to application (such as fluid lubricants stored within containers).

- Dwell and In-Flight Storage Effects-non-operational periods following some degree of shearing, typically without separating the contacting surfaces and often, but not always, in a vacuum (e.g., during in-flight dormant periods).

This review presents recent experimental data generated by ESTL into the potential effects of the above on the tribological performance of solid and fluid lubricants, together with insights gleaned from over 40 years of consultancy and testing support to the European space mechanisms industry.

As a general statement, little explicit data exists into the effect of long-term storage on spacecraft lubricants, and as a result, there are no industry-established software tools or models that can be employed. Data generated during long-term storage investigations produce, by its very nature, a sample size that is unlikely to be adequate for Weibull analysis (maximum likelihood estimators and fitting of degradation models). Likewise, studying thermo-kinetic models of any observed degradation chemical processes is hampered by the small sample size and the absence of an extensive array of measured properties. Therefore, one must draw from available datasets to gain confidence in the impact of these factors.

These phenomena must be adequately understood as lubricant degradation during spacecraft storage or in-flight could be mission-compromising.

\section{Materials and Methodologies}

\subsection{Environmental Control}

Much of the motivation for the work described herein stems from concerns surrounding the creation and maintenance of suitable test (and storage) environments, and the subsequent impact of these environments on the tribological components within a spacecraft mechanism. It is frequently reported that the level of humidity control and precision of monitoring practically achieved, both in general and specifically when considering mechanism storage post-integration at spacecraft level is relatively poor, and this may contribute to the variation in behaviours often observed. The limitations of the most relevant methods of environmental control are outlined below. A general comment is made that, in the opinion of ESTL, test/storage environments are often not monitored with enough accuracy, nor sufficiently local to the points of tribological contact.

- Evacuation of the test chamber - the quality and level of the vacuum achieved within a testing chamber depend not only on the dimensions of the chamber itself, its contents and the pump system but also the prior history of the test chamber hardware both in terms of maintenance and chemical exposure. Furthermore, it is not normally possible to define an absolute operating pressure for the full duration of any tests, rather tests are defined by a threshold start pressure with the chamber pressure allowed to (usually) decrease at some uncontrolled rate after the test starts.

- Gas purging - not only does the quality of purge gases vary but the concentration of trace elements is often impossible to determine or control. Also, particularly for larger test setups, leaks of moist air into the test chamber are possible and not always appropriately mitigated, monitored or minimised. The progression of purge gasses into the tribological contacts of a mechanism, particularly where convoluted pathways or labyrinth seals are employed, must also be considered. Without integrated and direct purge lines implemented in the mechanism design from the start, 
transport of purge gas into the cavities within mechanisms may be relatively slow/ineffective meaning the actual local environment of the tribological contacts is not fully known.

- Laboratory/cleanroom air-specifications can allow for significant variation in environmental factors such as temperature and RH. This variation is inevitable as maintaining a tight tolerance on the environment of a cleanroom would be prohibitively expensive.

\subsection{Tribometers and Test Facilities}

Tribological test activities described within this review were performed using either a Pin-on-Disc (PoD) or Spiral Orbit Tribometer (SOT). The differences and relative merits of each form of tribometer have been discussed in various literature [1,2], but the simplest consideration is to state that the PoD operates under predominantly sliding contact, whilst the SOT operates under predominantly rolling. Dependent upon the exact nature of the end application and the selected lubricant, either a tribometer may be more appropriate for a given study. Angular contact bearing tests were also performed to support certain testing activities.

\section{Operational Environment}

\section{1. $\mathrm{MoS}_{2}$ Operation with Environment}

It is well known that the tribological properties of certain lubricants are highly dependent upon the operational environment. Sputtered $\mathrm{MoS}_{2}$, for example, is known to display significantly higher friction coefficient and increased wear rates when operating in moist air due to rapid degradation of the thin film at the exposed boundaries of the S-Mo-S layers, caused by the presence of moisture and oxygen acting to degrade the low shear strength properties of the film. Whilst there are many reactions involving $\mathrm{MoS}_{2}$, oxygen and water vapour, two seem energetically most favoured.

$$
\begin{gathered}
2 \mathrm{MoS}_{2}+4 \mathrm{H}_{2} \mathrm{O}+\mathrm{O}_{2}=2 \mathrm{MoO}_{3}+4 \mathrm{H}_{2} \mathrm{~S} \\
2 \mathrm{MoS}_{2}+4 \mathrm{H}_{2} \mathrm{O}+9 \mathrm{O}_{2}=2 \mathrm{MoO}_{3}+4 \mathrm{H}_{2} \mathrm{SO}_{4}
\end{gathered}
$$

In ESTL's experience, reaction (2) takes place in condensing environments such as those of high humidity (not normal for most storage) in which the temperature fluctuates significantly. In these situations, the corrosive acidic product can damage the lubricant coating and for this reason, such environments can be particularly damaging for $\mathrm{MoS}_{2}$-lubricated components. Where the relative humidity and temperature are controlled, as in a cleanroom or in other kinds of on-ground storage, reaction (2) above does not appear to occur and instead, an oxide will form by the route given in equation (1) above. Of the above reactions, it is clear that (1), which occurs at a low rate under cleanroom conditions is the main concern for most credible mechanism/spacecraft level storage conditions.

However, the favourable properties of the lubricant under vacuum are known to be reversible, with the cycling of the test environment between air and vacuum allowing the performance of the lubricant to recover [3]. It is also known that some duration of in-air running, even if short, can have a profoundly detrimental effect on the subsequent life of the $\mathrm{MoS}_{2}$ coating under vacuum due to the increased wear rate of the lubricant film in the presence of moisture (Figure 1a) [4].

The tribological properties of sputtered $\mathrm{MoS}_{2}$ under $\mathrm{DN}_{2}$ are also not consistent with those achieved in a vacuum, with the lubricant displaying an extension in sliding lifetime under $\mathrm{DN}_{2}[5]$. Recent work performed by ESTL to support the Euclid mission [6] and by others [7] observe the same behaviour, with the latter describing this increase in durability as a consequence of beneficial contamination from the $\mathrm{DN}_{2}$ environments acting to improve the efficiency of the $\mathrm{MoS}_{2} 3^{\text {rd }}$ body lubrication. It is clear therefore that $\mathrm{DN}_{2}$ is not an accurate representation of vacuum for the operation of sputtered $\mathrm{MoS}_{2}$.

One question commonly asked is, "How moist is too moist for operation or storage?" European industry guidelines state that for solid lubricants (i.e., $\mathrm{MoS}_{2}$ ); 
- Prior to use, solid lubricated components should be stored in sealed bags purged with a dry inert gas (i.e., $\mathrm{DN}_{2}$ ).

- Any operational cycles should be performed under a $\mathrm{DN}_{2}$ atmosphere $(<15 \mathrm{ppm}$ moisture) as this is known to be "safe".

- Operation in environments with humidity levels above $5 \% \mathrm{RH}$ is known to cause $\mathrm{MoS}_{2}$ coatings to degrade i.e., the lifetime of the lubricant coating decreases

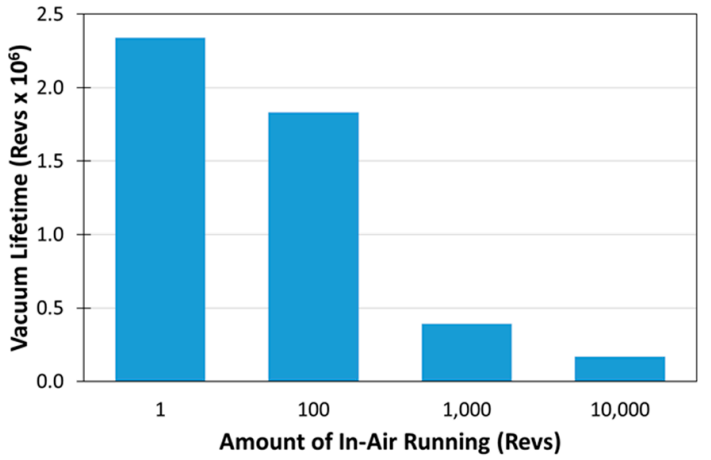

(a)

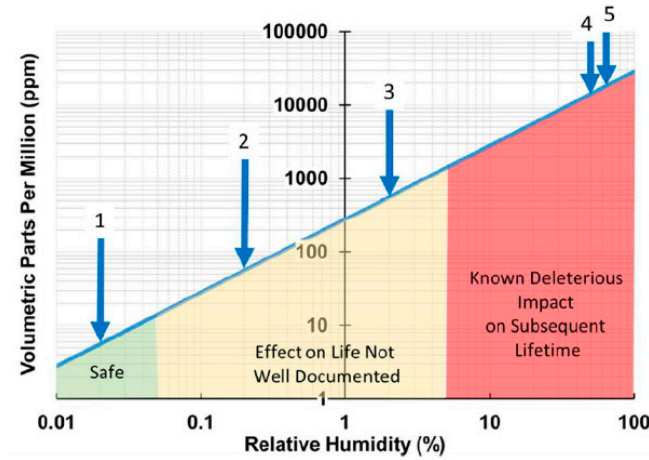

(b)

Figure 1. (a) Low-torque life of $\mathrm{MoS}_{2}$ lubricated bearings in a vacuum as a function of the amount of in-air operation prior to vacuum testing [4]; (b) regions of safe and deleterious impact on $\mathrm{MoS}_{2}$ lifetime showing assessment humidities [8]. Numbers indicate environments by [8].

However, much of the experimental evidence for these guidelines is limited and based on historical data which may no longer be accurate. In addition, the region of moisture between that defined as "safe" and "deleterious" is not well documented (Figure 1b) and, although this region of uncertainty should be avoided whenever possible, due to the issues of achieving dry conditions (described above) these environments may represent many practically achievable test conditions.

A PoD test campaign was performed to investigate the tribological performance of sputtered $\mathrm{MoS}_{2}$ within this hitherto poorly documented humidity region [8]. These tests suggest that in an environment of up to $2 \% \mathrm{RH} / \sim 500 \mathrm{ppm}$ moisture (achieved in $\mathrm{N}_{2}$ ), there is no evidence of a detrimental effect of water vapour on the tribological lifetime of sputtered $\mathrm{MoS}_{2}$ (Figure 2a). Above this value, the anticipated drop-off in lifetime is observed. This suggests that the deleterious effect of moisture does not occur until a moderately high $\mathrm{RH}$, and the statements for operation given above are somewhat conservative (Figure 2b).

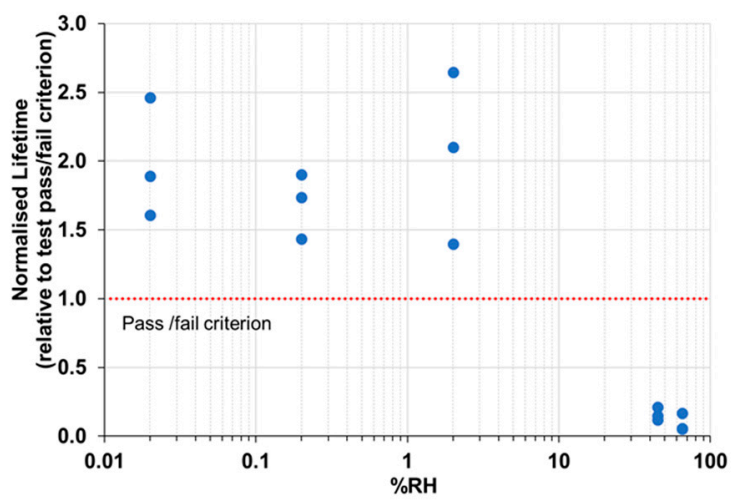

(a)

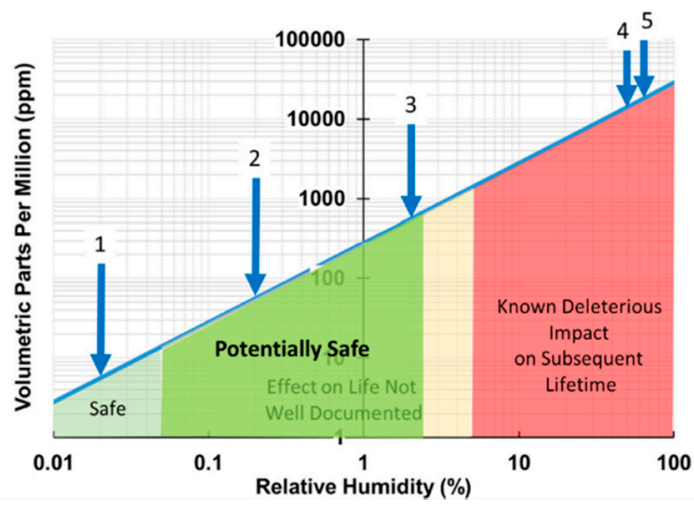

(b)

Figure 2. (a) Normalised lifetime of sputtered $\mathrm{MoS}_{2}$ vs. humidity (in $\mathrm{N}_{2}$ ). Performed at 1.5 GPa Peak Hertzian contact stress [8]; (b) New proposed regions of safe and deleterious impact on $\mathrm{MoS}_{2}$ lifetime following new data. 


\subsection{Some Period of Running in Moist Environments}

The impact of short duration (i.e., not fully to failure) of operation under these "safe" and "deleterious" environments on the subsequent vacuum lifetime of sputtered $\mathrm{MoS}_{2}$ has also been investigated. $\mathrm{MoS}_{2}$ coatings were sheared under $\mathrm{N}_{2}$ at a controlled moisture level for some predefined duration, before evacuating the chamber and allowing the test to continue to failure under vacuum.

Results showed that, for the "safe" region $\left(\leq 2 \% \mathrm{RH} \mathrm{DN}_{2}\right)$, the cumulative lifetime obtained of the sputtered $\mathrm{MoS}_{2}$ film ( $\mathrm{N}_{2}$ revolutions performed plus subsequent vacuum life) was consistent (Figure 3). This suggests that, when sufficiently dry, operation in nitrogen does not significantly influence the sputtered $\mathrm{MoS}_{2}$ beyond cumulative wear.

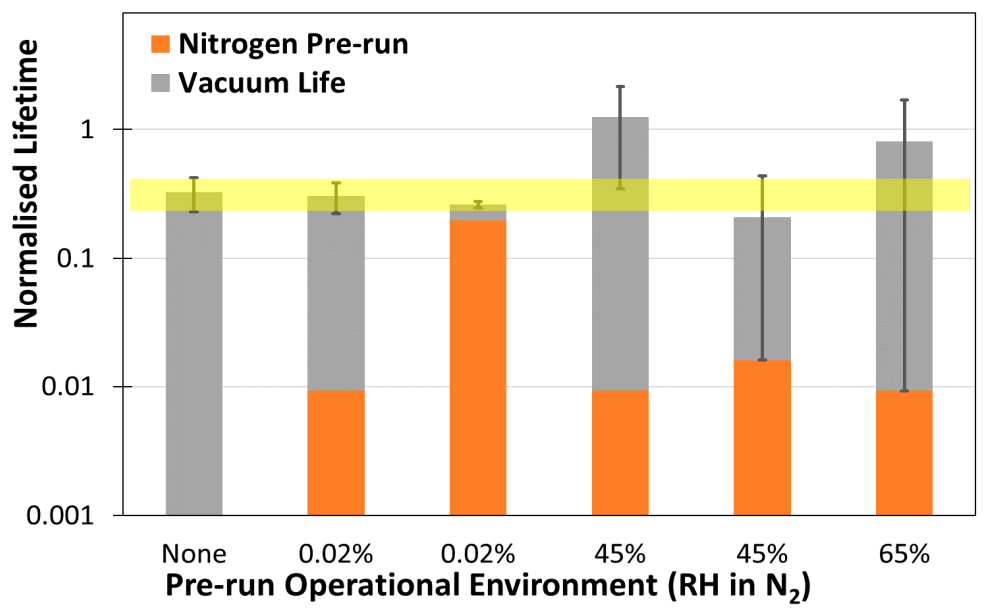

Figure 3. Normalised total sliding life of sputtered $\mathrm{MoS}_{2}$ following varying periods of $\mathrm{N}_{2}$ operation. Yellow bar indicates typical variance.

More interesting is the observation that, with prior shearing in moist $(>2 \% \mathrm{RH})$ environment, the cumulative lifetime of sputtered $\mathrm{MoS}_{2}$ is seen in increase in some instances. Observations of this extension in $\mathrm{MoS}_{2}$ life when exposed to moist environments have been made before [6,9,10] but were commonly attributed to test variability (amongst other things). However, given that this increased life appears to occur only at higher RH values, and an improved understanding of the nature of $\mathrm{MoS}_{2}$ variability on the PoD [10], this assumption should be re-evaluated. It is argued that the adage of exposure and/or shearing under a moist environment being universally detrimental to sputtered $\mathrm{MoS}_{2}$ is potentially too simplistic, and under some (as yet undefined) conditions, exposure to moisture may improve the subsequent in-vacuum lifetime of the lubricant. However, until better understood, it is still essential that the guidelines above be followed for the purposes of risk mitigation.

\subsection{Hybrid Lubrication to Mitigate Environmental Behaviours}

The suggestion is occasionally made within the space mechanisms community that a form of hybrid lubrication may circumvent the restriction on in-air operation of $\mathrm{MoS}_{2}$, through the application of a controlled quantity of PFPE fluid lubricant to a component lubricated in the conventional way with sputtered $\mathrm{MoS}_{2}$, thus protecting the $\mathrm{MoS}_{2}$ from the moist environment. The suggestion states that the fluid lubricant will provide low friction during operation in moist air and will subsequently be lost (through either evaporation or tribo-chemical degradation) under vacuum, allowing the (hopefully) uncompromised $\mathrm{MoS}_{2}$ film to provide low friction and long life for the remainder of operation under vacuum.

Feasibility trials have been performed using the SOT where 52,100 steel test balls were first lubricated with sputtered $\mathrm{MoS}_{2}$. These same test balls were then lubricated with $\sim 50 \mu \mathrm{g}$ of PFPE oil applied via solvent plating. Hybrid lubricated balls were inserted into the SOT test chamber and rotated in moist air for a defined duration and subsequently under a high vacuum to failure. Results 
demonstrated encouraging behaviour, with indications that a small volume of fluid lubricant acts to protect the sputtered $\mathrm{MoS}_{2}$ from elevated wear rates in moist air. In addition, the friction coefficient of the hybrid lubrication was found to be $\sim 0.02$ (Figure 4), identical to the value for $\mathrm{MoS}_{2}$ alone in vacuum [2]. Given that Z25 provides a friction coefficient of $\sim 0.1$ under vacuum on the SOT [2], it can be said with some confidence that the $\mathrm{MoS}_{2}$ was providing lubrication at this time and had been protected from a reasonable degree of moist air-operation.

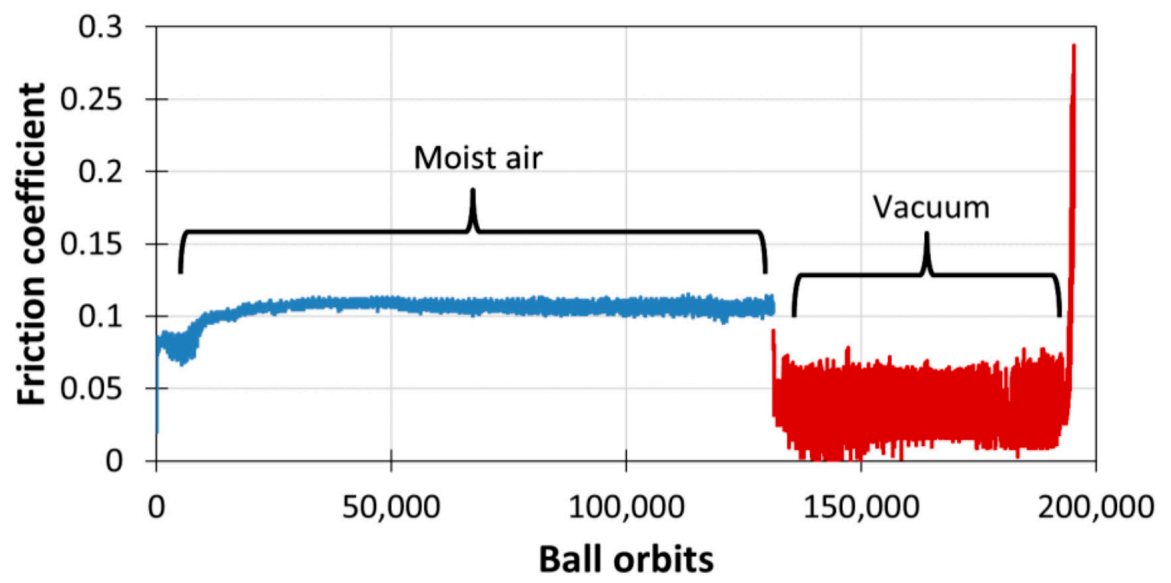

Figure 4. Hybrid PFPE/MoS 2 rolling in air (blue) and vacuum (red). Elevated frictional noise during vacuum running is an artefact introduced by the analysis software and is not real.

Furthermore, under ideal conditions (believed to be dependent upon the physical properties of the fluid, such as viscosity and film thickness [11]), the lifetime of a sputtered $\mathrm{MoS}_{2}$ film can be significantly extended under vacuum, with no consequential increase in friction coefficient, through the application of a fluid film. Component- and tribometer-level research activities are continuing at ESTL to verify this hypothesis.

\subsection{Lead Operation with Environment}

Little experimental evidence exists as to the impact of the operational environment on thin lubricating films of lead. [12] states that an oxide layer of lead does not adversely influence the friction coefficient of the bulk material, but SOT activities performed by ESTL show clear differences in the tribological properties of the lubricant when rolling under vacuum and laboratory air. The friction coefficient of lead under air on the SOT is measured as $\geq 0.13$, in comparison to $\leq 0.05$ under vacuum, with a lifetime of $\sim 10^{6}$ orbits in the air (typically $2 \times 10^{7}$ achieved under vacuum).

In addition, exposure to in-air heating (up to 8 -h at $200{ }^{\circ} \mathrm{C}$ ) has also been shown to increase the start-up rolling friction, reduce the rolling lifetime, and increase the run-in duration required to achieve low friction on the SOT [13]. If we assume that the reaction at the coating surface is purely oxidation, and the moisture content of the environment remains the same (a simplification), then a brief period of in-air heating can be equated to a longer period of in-air exposure at ambient periods. With this logic, the above observations for lead are similar to those for $\mathrm{MoS}_{2}$, where periods of in-air exposure are known to temporarily increase the friction coefficient of the lubricant under vacuum [3].

\subsection{Fluid Lubricant Performance with Environment}

It is often assumed that the tribological behaviour of fluid lubricated components will be insensitive to the operational environment, but in many instances, this may be a simplification. Experimental investigations conducted using the SOT show that the tribo-consumption rate of PFPE oil is greatly affected by the presence of small quantities of water vapour [14]. The degradation rate of PFPE [15] and MAC lubricants [16] are also known to be influenced by surface cleanliness, leading to the suggestion that the presence of water vapour (or other contaminants such as an oxide layer) on the substrate 
surface acts to retard the degradation rate of vacuum fluid lubricants. Under shear, the native oxide layer present on the surface of the substrate steel is removed, revealing the nascent metal. This nascent steel is much more reactive than the original oxide layer, resulting in accelerated chemical degradation of the fluid.

In an ambient air environment, and if oxidation occurs fast enough, this oxide layer can be replenished, retarding the degradation rate of the fluid. This has been experimentally demonstrated in PoD (sliding) and SOT (rolling) investigations, where the lubricating lifetimes of a PFPE and MAC-based grease (Braycote 601EF and MAPLUB SH100-b) were found to be significantly higher in an ambient air environment (Figure 5a), with marginally lower friction coefficients [17]. PFPE lubricants have also been shown to provide extended lifetimes when operating in a modest vacuum $\left(10^{-2}\right.$ bar) [8]. Assuming that the residual pressure within a vacuum chamber is predominantly moisture released from the internal surfaces, this correlates well with the consideration that the presence of moisture results in increased lubricant lifetimes.

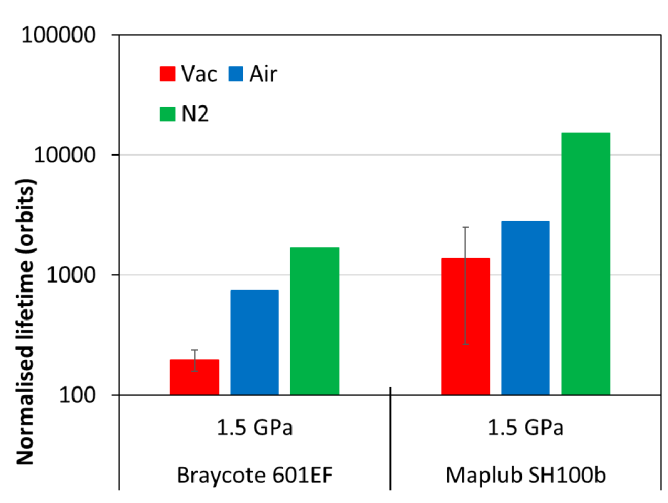

(a)

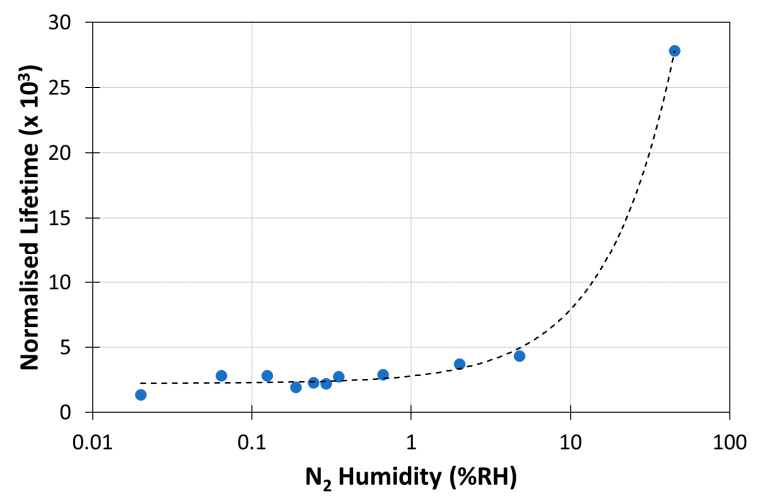

(b)

Figure 5. (a) Normalised lifetime (orbits/ $\mu$ g) of Braycote 601EF and Maplub SH100b grease under various environments as assessed on the Spiral Orbit Tribometer (SOT) [17]; (b) Normalised lifetime (orbits/ug) of PFPE oil (Fomblin Z25) with varying humidity in $\mathrm{N}_{2}$ (with linear extrapolation) [8].

Curiously the lifetimes obtained via operation in $\mathrm{DN}_{2}$ are also increased (Figure 5a). Whilst it is clear that the humidity content within a nitrogen environment impacts upon the lifetime of PFPE lubricants (Figure 5b), it is also clear that the moisture alone cannot be the sole cause of this increase in life. This can be further demonstrated via the observation of increased rolling lifetimes of PFPE fluids in moist nitrogen, when compared with ambient air of equal RH [8]. The influence of oxygen has been suggested as a potential explanation for this behaviour.

These observations lead to the interpretation that as long as the expected friction/torque differences are considered, on-ground operations of fluid lubricated components in non-vacuum environments can be considered "safe"; i.e., they will not result in compromised behaviour of the lubricated component during subsequent vacuum operation. However, the above data suggests also that, IF the target is to test on the ground with minimal cumulative tribo-chemical degradation, one would be better performing the test in a moist $\mathrm{N}_{2}$ (say $45 \% \mathrm{RH}$ ) environment, rather than in moist air.

\section{On-Ground Storage Behaviours}

In addition to the operational environment, attention must be given to pre-operational storage conditions for lubricated components. Storage conditions are ideally selected on the assumption that the environment is benign, but often this has not been explicitly demonstrated. 


\subsection{Maturation of Sputtered $\mathrm{MoS}_{2}$}

Recent experimental investigations suggest that sputtered $\mathrm{MoS}_{2}$ may "mature" upon storage, such that the tribological properties of freshly deposited $\mathrm{MoS}_{2}$ differ following some period of storage. This has been investigated in detail by ESTL where a series of PoD tests were performed over a period of 60 days on a single batch of sputtered $\mathrm{MoS}_{2}$ lubricated 52100 -steel discs. Test coupons were stored in $\mathrm{DN}_{2}$ prior to the assessment.

Results demonstrated a marked increase in lubricant lifetime (but also some increased lifetime variance) as storage duration increases, suggesting that exposure to the commonly considered benign $\mathrm{DN}_{2}$ acts to alter the lubricant performance. A parallel test campaign performed on the SOT displayed the same behaviour, demonstrating that this maturation effect is not unique to the sliding motion of the PoD tribometer. Steady-state friction coefficient did not show a strong correlation with the storage environment, and no relationship between steady-state friction and lifetime was observed during these tests. Little evidence was seen of a correlation between coating thickness and tribological performance, in agreement with previous observations [15].

A follow-up campaign of PoD tests was performed by ESTL under identical conditions, with sputtered $\mathrm{MoS}_{2}$ lubricated samples stored for up to 12-months (in $\mathrm{DN}_{2}$ and laboratory air). Results from this experiment showed evidence of increasing lubricant lifetime over the first 60 days, followed by a plateau in which the tribological behaviour (including lifetime) was unchanged.

A final phase of the experimental campaign was performed utilising "opportunistic" lubricant samples from ESTL's own $\mathrm{MoS}_{2}$ long-past production activities. $\mathrm{MoS}_{2}$ test coupons from production dating back some 16-years and exposed in the intervening time to uncontrolled (i.e., room air) storage were assessed via $\mathrm{PoD}$, and their tribological performance compared against their original behaviour at the time of production. These lubricated coupons showed no statistically significant change in coating lifetime as a result of such storage.

Taken together these experimental investigations provide evidence of a "maturation" effect of $\mathrm{MoS}_{2}$. The initial phase of this maturation appears to occur over a few months following the deposition of the coating, before plateauing and maintaining consistent tribological behaviour beyond this point, implying some level of passivation-type behaviour. The exact details of how this maturation occurs are not known but may be related to the observation from [7] that the presence of contaminants (including $\mathrm{H}_{2} \mathrm{O}, \mathrm{CO}_{2}, \mathrm{O}, \mathrm{N}_{2}$ and $\mathrm{H}_{2}$ ) within an operational environment is favourable for the $\mathrm{MoS}_{2}$ lubrication.

\subsection{Long Term Storage of Fluids}

Fluid lubricants are particularly susceptible to effects of on-ground storage which may influence their tribological properties. These include oxidation effects which have the potential to change the viscosity of lubricants over time (particularly MAC-based oils), absorption of moisture, loss of additives, and chemical degradation as well as physical effects such as base oil separation (for greases) and lubricant creep. As a result, fluid lubricant manufacturers often provide guidelines for lubricant storage (often at low temperatures) and shelf lives of typically 5yrs. However recent SOT testing performed by ESTL on historical PFPE oils, some stored under uncontrolled conditions (i.e., room air) for 40-years, have shown similar tribological behaviour to new as-formulated PFPE oils, with no reduction in lubricating lifetime. This indicates that there may be no significant effect of uncontrolled air storage on the tribological performance of PFPE oils.

\subsection{Phenolic Cages}

In many applications of fluid lubrication, bearings are fitted with cotton fabric reinforced phenolic cages (retainers). It is common practice to vacuum-impregnate such cages with the base-oil of the fluid lubricant used in the bearings to saturate the cotton fabric with the lubricant oil prior to use (and inhibit the uptake of the free oil within the bearing). There is however evidence [18] that even "fully impregnated" cages when continuously wetted by further exposure to oil may absorb a significant 
additional oil mass as the oil is gradually taken up by the phenolic matrix itself. It can be shown that the typical capacity of the cage for this additional oil uptake, represents a disturbingly high percentage of available free oil. For example, the infiltration of oil into the phenolic matrix is a slow process, taking months, but as shown in Figure 6 the total mass absorbed can be relatively high. Assuming this oil is absorbed entirely from the free oil quantity typically added to a bearing, the proportion of oil taken-up can quickly reach $100 \%$. This phenomenon is potentially an overlooked lubricant loss route which may cause the onset of starvation/dry running within a bearing.

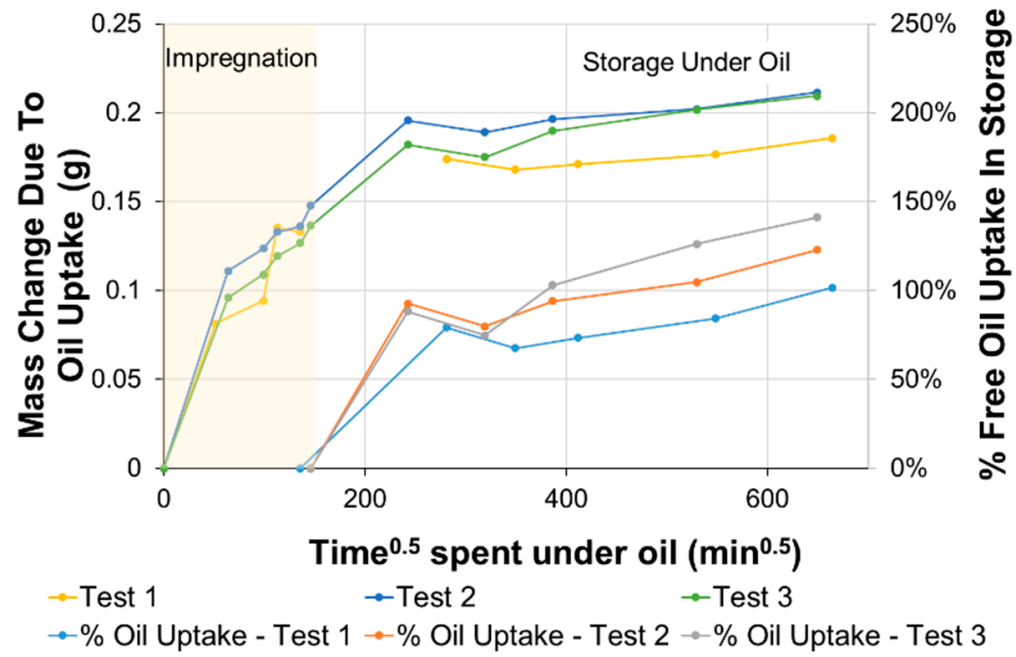

Figure 6. Oil mass uptake by weight (left axis) and potential impact on the proportion of free oil (Basis: $28 \mu \mathrm{L}$ free oil available, storage period up to 9 months) (right axis) [18].

\subsection{Creep Barriers}

Creep barriers are commonly used to mitigate and control the unwanted creep of lubricant within a fluid lubricated application. Shelf lives for creep barriers are often quoted as 1 year, and Minimum Order Quantities (MOQ) can be, relative to the quantity needed, prohibitively large (e.g., MOQ 5 kg). Product lead-times may also be long, and as a result, it is common that a creep barrier intended for an application may be formally borderline "life-expired" or even fully beyond its quoted shelf life when required for use. In order to verify whether the performance of such formally expired creep barriers remains acceptable for a flight application, a re-lifing process must be completed, with a series of tests performed and the results compared against the desired/known behaviour of the in-life product. These tests should include an optical inspection if the creep barrier fluid, a check of the UV marker (if the creep barrier contains one), surface energy assessment (i.e., via contact angle), crosshatch adhesion test, and FTIR analysis.

\subsection{Corrosion}

Substrate corrosion can occur in the presence of a condensing environment. This may be caused by residual contamination (e.g., chlorides) or by the acidic by-product of the reaction between $\mathrm{MoS}_{2}$ and moisture in a condensing environment (Reaction (2) above). This can initiate corrosion of the substrate and is relevant to components lubricated with PVD MoS 2 and self-lubricating polymers that contain $\mathrm{MoS}_{2}$ which may be exposed to a condensing environment. The speed of this reaction is a function of temperature. In a non-condensing environment, the oxidation of $\mathrm{MoS}_{2}$ (Reaction (1), above) is limited to a very thin outer surface layer ( 100 Angstroms) and the reaction product is non-acidic, meaning that in the absence of other reacting species, corrosion is unlikely to be an issue. Substrate corrosion is typically identified by detailed visual inspection.

In addition, there is the risk that, in the presence of a moist environment, corrosion is initiated between metals with highly different electrochemical potentials. For example, mechanisms having 
several different metallic interfaces (e.g., from industry a threshold of $>0.25 \mathrm{~V}$ electro-chemical potential difference is taken as a cause for concern) in local contact (bearings, housings, end-caps and clamp rings for example) could be at risk from galvanic corrosion. In this case, a representative "equivalent assembly" or witness sample subassembly may be more useful for assessing the impact of storage.

\subsection{Seals Under Loading}

Creep under load of polymeric seals (O-rings) has been reported as a concern by the recent European spacecraft programmes. This may be quantified at the component level by a load vs. deflection measurement made as a sealed interface is offloaded.

\section{Dwell Effects and In-Flight Storage}

In-flight operation and storage (i.e., prolonged in-vacuum stasis prior to operation-as for example in robotic devices for planetary missions or in devices required for de-orbiting spacecraft at end-of-mission) will bring its own considerations, challenges and mitigations. Where possible for fluid-lubricated mechanisms, the orientation of the spacecraft or mechanism should be selected to minimise its average temperature during the storage period. This is due to lubricant creep, degradation (including due to potential autocatalysis of PFPEs), and the evaporation rate of oils all displaying strong dependencies upon temperature. This measure will inhibit most degradation phenomena and maintain the fluid lubricant within the mechanism to the maximum extent - but may not always be feasible for spacecraft operational reasons.

For in-flight storage, the environment can be highly variable dependent on the mission profile. Whilst typically high/ultra-high vacuum and ranging from, say -40 to $+80{ }^{\circ} \mathrm{C}$, the environment for in-flight storage can also encompass more extreme thermal excursions for exposed items. For mechanisms on different types of science missions a full range from $\sim 20 \mathrm{~K}$ to $\sim 600 \mathrm{~K}$ and environments including $\mathrm{CO}_{2}, \mathrm{H}_{2}$, traces of $\mathrm{CH}_{4}$ or, for $\mathrm{LEO}$, atomic oxygen could be relevant depending on mechanism location and mission type.

The vacuum level in GEO is typically of order $10^{-13}$ mbar, however, near a satellite, the pressure is higher, about $10^{-10}$ mbar, as a result of outgassing from the satellite body. Within the spacecraft itself the pressure is yet higher. Calculations have shown, for a Solar Array Drive Mechanism (SADM) for example, that the internal pressure reaches $\sim 10^{-5}$ mbar soon after orbital height is attained and decreases during the first year of operation to about $10^{-7} \mathrm{mbar}$ (comprising $~ 80 \%$ water vapour and $\sim 20 \%$ carbon monoxide) depending on the balance between the pump-out speed of venting orifices and declining outgassing rates [19]. Furthermore, in the case of reaction wheels there is the possibility of in-flight storage as well as operation under a partial vacuum (believed to be typically around $\left.10^{-3} \mathrm{mbar}\right)$.

\subsection{Evaporation Effects}

Over a long duration storage period, the loss of fluid lubricant through evaporation must be considered. This is particularly true in instances where storage temperature is elevated for some or all of the storage period (of greater concern during in-flight storage than on-ground). Such evaporation is generally controlled through labyrinth sealing.

Losses due to evaporation can be estimated using the Langmuir equation approach. However, in general, a lubricant may have components of different molecular weights and a range of volatilities. This can render analysis using the Langmuir equation and published vapour pressure data too simplistic, the true situation is that the rate of mass loss, even for nominally single component fluids without additives is dependent on the thermal history of the lubricant. Indeed, there is evidence [18] that the Langmuir equation may predict higher loss rates than found in practice by approximately an order of magnitude at typical operating temperatures. 


\subsection{Fluid Separation and Creep}

Concern exists regarding the separation of the oils (also known as "oil bleed") from the grease, allowing migration to other surfaces. This can cause changes in the consistency of the grease and in so doing may alter its tribological properties. In the extreme, it may create lubrication-starved conditions during initial operations. Oil separation usually occurs on static components and is thus likely to occur during prolonged storage. It is considered that when using oils and greases in long-term applications or when mechanisms are subjected to long periods of storage, it is advisable to run the unit in question periodically to redistribute the lubricant if there has been pooling of oil due to oil separation or slumping due to storage conditions and orientation - implicitly this suggests such periodic "exercising" can locally re-mix oil back into the grease. In this respect, the use of creep barriers is also important as it restricts the surface migration of oil from the lubricated area. Without such barriers, oil could be lost irretrievably during prolonged storage from lubricated areas.

Where a small quantity of oil is used, 1-g migration is minimised as the lubricant tends to remain within the contact and on the surfaces of the component, due to surface energy effects. This is true for relatively small quantities of oil, of order $\sim 1 \%$ to $<\sim 5 \%$ free-volume fill (i.e., this amounts typically to $\sim 1 \mu \mathrm{L} / \mathrm{mm}$ bearing PCD). However, for larger volume fills, as might be used if viscous losses are less a concern or total evaporative losses over the mission duration expected to be high (due to, for example, high-temperature operation and/or long lifetime), migration in 1-g is a much more likely phenomenon as the mass of the fluid body is high compared to the retaining viscous forces and substrate oil-film surface energetics. Such 1-g migration may impact the subsequent flight lifetime of the mechanism if the oil is able to exit the bearing envelope.

During on-ground storage creep is driven by surface energy differentials and also by gravity, and can be minimised partly by control of surface features (less likely on smooth surfaces, or to migrate around sharp edges) and (primarily) by the appropriate application of anti-creep coatings which have a lower surface energy than the lubricating fluid.

\subsection{Dwell Behaviours of Solid Lubricants}

The influence of in-situ (vacuum and air) dwell periods on the subsequent in-vacuum tribological properties of PVD $\mathrm{MoS}_{2}$ and lead has been investigated at PoD and angular contact bearing level [20].

When assessed in pure sliding, exposure to an in-air dwell period acted to increase the start-up friction coefficient of sputtered $\mathrm{MoS}_{2}$. The rate of increase in friction with exposure time appears to level off at exposure periods of $10^{5} \mathrm{~s}$, demonstrating a saturation effect. Steady-state friction values are unaffected by in-air dwell periods, but the recovery period (i.e., the number of disc revolutions required to re-establish low friction in vacuum) increases logarithmically (Figure 7).

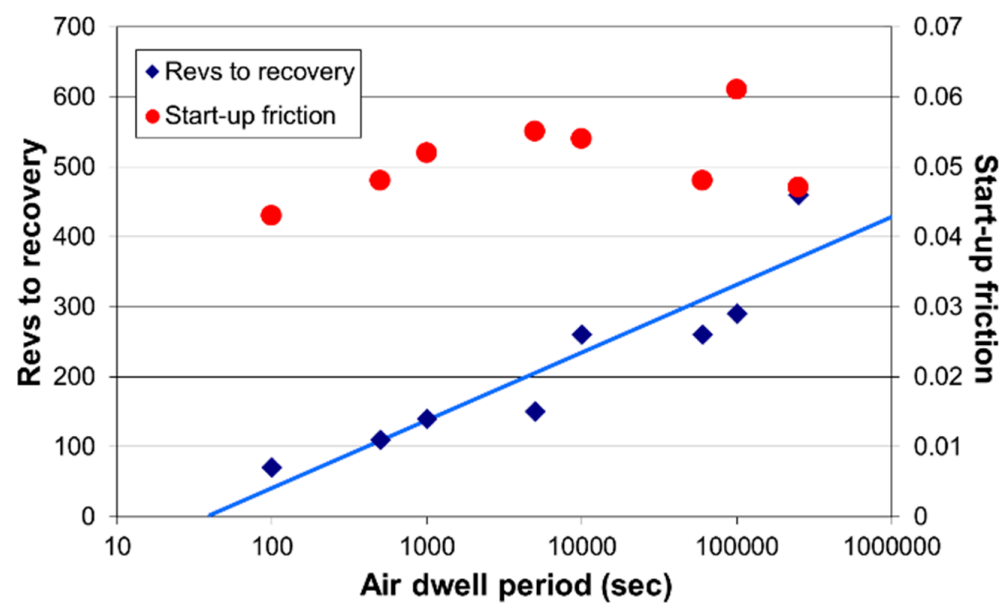

Figure 7. Start-up friction and revs to recovery under vacuum of sputtered $\mathrm{MoS}_{2}$ following in-air dwell (data shows 1.50 GPa Peak Hertzian contact pressure [20]. 
$\mathrm{MoS}_{2}$ lubricated bearings (fitted with PGM-HT cages) displayed higher start-up torque in vacuum as a result of periods of dwell in both laboratory air and vacuum environments [20]. The most significant increase in torque was a factor of $\sim 2.75 \times$ higher than the run-in torque (i.e., less than the equivalent effect observed under a pure sliding motion but potentially significant in relation to mechanism torque margins), occurring after an exposure period of $10^{6} \mathrm{~s}$ in air. The number of revolutions required to recover low torque also increased with increasing dwell time, in a similar manner to that seen at the PoD level.

After being correctly run-in in vacuum, PVD lead-lubricated bearings display no measured effects of dwell, either in vacuum or laboratory air, up to $10^{6} \mathrm{~s}$.

\subsection{Auto-Catalytic Degradation}

Perfluorinated oil-based fluid lubricants such as the grease Braycote 601EF or Fomblin Z25 oil are highly susceptible to chemical degradation, whereby a reaction occurs between the fluorine released from the oil by the action of shearing and iron within the substrate steel [21]. This reaction produces the compound $\mathrm{FeF}_{3}$ which further increases the rate of fluid degradation.

This reaction acts to destroy the long-chain polymers within the fluid, removing the attractive low shear properties of the fluid, causing a rapid increase in friction coefficient and ultimately restricting the lifetime of these lubricants. Although usually understood to occur during operation (i.e., shearing), it has been postulated that once this reaction has been initiated through the shearing of the fluid (for example during mechanism run-in, acceptance test or initial in-flight commissioning), degradation could potentially continue when the fluid is not being sheared due to the existing presence of FeF 3 within the contacts [22]. Such behaviour is referred to as the Autocatalytic Effect (ACE) and could potentially cause continued degradation of PFPE lubricants, following initial shearing, when stationary (e.g., degradation potentially continuing during both ground storage and during periods of in-flight inactivity, such as for missions having a long cruise phase). The concern is that such behaviour could impact the overall lubricant lifetime or produce significantly higher torques upon restart of a mechanism following an extended dwell period either on the ground or in flight.

Despite its theoretical feasibility, to date there is limited experimental evidence of auto-catalytic degradation of a PFPE lubricant from tribometer testing. Numerous experimental PoD and SOT campaigns performed by ESTL have been inconclusive in observing this effect in all instances beside those where test conditions were selected to be deliberately harsh and designed to induce autocatalytic degradation [22]. Nevertheless, ACE remains a concern, albeit theoretical, for fluid lubricated mechanisms.

\subsection{Periodic Actuation/Exercising}

At the mechanism level, general practice is to always maintain mechanisms intended for on-ground storage in a dry nitrogen purge and to occasionally "exercise" the mechanism. The term exercise is used to describe the periodic operation on the ground for a low number of cycles in order to recover pre-storage levels of performance, or to detect incipient storage issues at a time when preventative action or rectification measures may be implemented.

Whilst the requirement to exercise a mechanism during storage is usually taken as being most applicable to grease or oil lubricated devices for which the exercise operations may re-distribute or replenish the local supply of oil or grease within the tribo-contacts (discussed above), it should also be noted that limited exercising may also be appropriate for solid lubricated mechanisms in order to avoid or minimise the impact of localised lubricant film indentations occasionally reported due to lubricant creep within preloaded contacts. In addition to these effects local to preloaded, lubricated tribo-contacts, consideration must also be given to the avoidance or potential impact of preload spring (or diaphragm) stress-relaxation during storage (an early life effect which can be designed-out).

Whilst it is conventional to calculate the required qualification lifetime test duration based upon foreseen on-ground and in-orbit operational cycles, it should be noted that any additional operational 
cycles required as a result of the need to carry out the periodic exercising of a device during long-term storage on-ground must preferably also be included (and appropriately factored) in the calculation of qualification lifetime. However, this may be practically difficult in some circumstances as the need for long-term storage cannot always be anticipated, it being potentially required due to prior launch failures or other unforeseen circumstances occurring after qualification and delivery of flight hardware. The frequency and duration of exercise operations during ground tests need to be considered carefully. The following considerations are applicable for different applications:

- For some applications, a few revolutions or cycles may represent a significant proportion of the in-flight lifetime. In these cases, the duration should be considerably reduced and may ultimately be replaced by small oscillations through an angle large enough to provide overlap of ball positions on bearing raceways (so preventing local lubricant build-up).

- In other applications, operation in dry nitrogen or in air for a prolonged duration may generate excessive particulate debris or lubricant degradation which could compromise subsequent performance (e.g., for lead lubrication operated cumulatively beyond $10^{5}$ cycles in air, or for $\mathrm{MoS}_{2}$ where even a few revs in the air can greatly impact subsequent in-vacuum lifetime [4]). Therefore, the exercise duration (and environment) should be carefully chosen to minimise such impacts.

- In some applications, exercising the device will have some unique and potentially high risks (e.g., requiring de-integration from a spacecraft) which need to be weighed against the benefits obtained from the exercise regime.

Therefore, the main consideration for exercising a device is to perform the minimum cycles to mitigate any storage phenomena and confirm beyond doubt that the unit is in-family with its pre-storage performance. Tribological devices exhibit a range of behaviours depending on the environment, previous test history and many other factors, however, the aim of the mechanism exercise regime is to achieve, upon completion of each exercise event, in-family, in-specification and stable behaviour considering the test environment. This should be close to the behaviour at the commencement of storage assuming the same setup and test conditions.

\section{Conclusions}

The increased interest in interplanetary, lunar and other robotic missions, combined with the need to employ mechanical systems for spacecraft de-orbit or in-orbit servicing seems likely to drive the need for a better understanding of in-orbit storage phenomena. Furthermore, some recent programmes in which the economies associated with batch-builds of sequences of satellites, as well as the occasional un-planned launch delays dictate that a better understanding is required of on-ground storage effects and the potential mitigations. Although some storage best practice guidelines exist (including those discussed above) to date little experimental verification has been performed. Therefore, many concerns exist which cannot easily be allayed by experimental data, indeed where experimental data does exist, this is often "opportunistic" and may provide quite limited statistical confidence.

All space lubricants are tribologically susceptible to environmental effects during operation and storage, but with widely differing degrees of severity. As a general statement, the above experimental investigations show that it is important, whenever possible, to operate both solid and fluid lubricated mechanism components under environments representative of their intended application. In addition, the monitoring and understanding of storage and operational environments is extremely important to gain confidence in the tribological performance of spacecraft mechanism components to be used in future ambitious missions.

Author Contributions: M.B. is the main author of the paper with inputs, contributions, and reviews by S.L., A.K. and R.B. Experimental activities, analysis, inspections and data interpretation was performed by all authors. All authors have read and agreed to the published version of the manuscript.

Funding: This research was funded in part by ESA-ESTEC. 
Acknowledgments: This review paper discusses research activities and programs performed by many staff at the European Space Tribology Laboratory, past and present. Additionally, inputs and guidance towards the research was provided by the ESA-ESTEC TEC-MSM team. The authors gratefully acknowledge this support.

Conflicts of Interest: The authors declare no conflict of interest.

\section{References}

1. Pepper, S.V.; Kingsbury, E.P. Spiral Orbit Tribometry-Part I: Description of the Tribometer. Tribol. Trans. 2003, 46, 57-64. [CrossRef]

2. Buttery, M. An Evaluation of Liquid, Solid, and Grease Lubricants for Space Mechanisms Using a Spiral Orbit Tribometer. In Proceedings of the 40th Aerospace Mechanisms Symposium, Cocoa Beach, FL, USA, 12-14 May 2010; pp. 59-71.

3. Roberts, E.W. The Tribology of Sputtered Molybdenum Disulphide Films. Tribol. Frict. Lubr. Wear Fifty Years IMechE 1987, 1, 503.

4. Cunningham, J.M. Effects of Ground Testing on The Torque Performance of MoS2 Lubricated Ball Bearings Fitted with Duroid Cages; ESA-TM-193; ESR Technology: European Space Tribology Laboratory: Warrington, UK, 1998.

5. Gardos, M.N. Anomalous Wear Behaviour of $\mathrm{MoS}_{2}$ Films in Moderate and Dry Nitrogen. Tribol. Lett. 1995, 1, 67-85. [CrossRef]

6. Buttery, M.; Stanley, S. PoD Tests to Support Euclid Program; ESA-ESTL-TM-0211; ESR Technology: European Space Tribology Laboratory: Warrington, UK, 2016.

7. Colas, G.; Saulot, A.; Michel, Y.; Baudasse, Y.; Mistral, A.; Berthier, Y. Dry Lubrication Efficiency: From Ground to Space. In Proceedings of the 15th European Space Mechanisms and Tribology Symposium, Noordwijk, The Netherlands, 25-27 September 2013.

8. Buttery, M. Effect of Relative Humidity on Lubricant Performance; ESA-ESTL-TM-0219; ESR Technology: European Space Tribology Laboratory: Warrington, UK, 2019.

9. Buttery, M.; Roberts, E.W. Impact of Environment on $\mathrm{MoS}_{2}$; ESA-ESTL-TM-0118; ESR Technology: European Space Tribology Laboratory: Warrington, UK, 2014.

10. Buttery, M.; Kent, A. Understanding of $\mathrm{MoS}_{2}$ Test Variation (Phase 1); ESA-ESTL-TM-0218; ESR Technology: European Space Tribology Laboratory: Warrington, UK, 2018.

11. Buttery, M.; Kent, A.; Forster, D.; Vortsellas, A. Hybrid Lubrication of PFPE Fluids and Sputtered $\mathrm{MoS}_{2}$. In Proceedings of the 44th Aerospace Mechanisms Symposium, Cleveland, OH, USA, 16-18 May 2018.

12. Clauss, F.J. Solid Lubricants and Self-Lubricating Solids, 1st ed.; Academic Press: New York, NY, USA, 1972.

13. Buttery, M. Effect of Curing Temperature on Solid Lubricant Films; ESA-ESTL-TM-0081; ESR Technology: European Space Tribology Laboratory: Warrington, UK, 2011.

14. Pepper, S.V. Effect of Test Environment on Lifetime of Two Vacuum Lubricants Determined by Spiral Orbit Tribometry. In Proceedings of the 38th Aerospace Mechanisms Symposium, Pasadena, CA, USA, 17-19 May 2006.

15. Buttery, M. Spiral Orbit Tribometer Assessment of Space Lubricants; ESA-ESTL-TM-0066; ESR Technology: European Space Tribology Laboratory: Warrington, UK, 2010.

16. Pepper, S.V. Lifetimes of Multiply Alkylated Cyclopentane Oil in Contact with Various Metals Evaluated with Vacuum Spiral Orbit Tribometer; NASA/TM-2018-219869; NASA Glenn: Cleveland, OH, USA, 2018.

17. Kent, A. Lifetimes of Space Grease in Air; ESA-ESTL-TM-0190; ESR Technology: European Space Tribology Laboratory: Warrington, UK, 2016.

18. Lewis, S.; Buttery, M.; Poyntz-Wright, O.; Kent, A.; Vortsellas, A. Accelerated Testing of Tribological Components-Uncertainties and Solutions. In Proceedings of the 44th Aerospace Mechanisms Symposium, Cleveland, OH, USA, 16-18 May 2018.

19. Robbins, E.J. Tribology Tests for Satellite Applications: Simulation of the Space Environment. In Proceedings of the 1st European Tribology Symposium, Frascati, Italy, 9-11 April 1975; pp. 101-113.

20. Buttery, M.; Cropper, M. Effect of Air Exposure and In-Vacuo Dwell on $\mathrm{MoS}_{2}$ and Pb; ESA-ESTL-TM-0071; ESR Technology: European Space Tribology Laboratory: Warrington, UK, 2010. 
21. Mori, S.; Morales, W. Tribological Reactions of Perfluoroalkyl Polyether Oils with Stainless Steel Under Ultrahigh Vacuum Conditions at Room Temperature. Wear 1989, 132, 111-121. [CrossRef]

22. Buttery, M.; Gaillard, L.; Rajala, S.; Roberts, E.; Rohr, T.; Merstallinger, A. Fomblin Z25: A New Method for its Degradation Assessment \& Proposal for Safe Operation in Space. In Proceedings of the 15th European Space Mechanisms and Tribology Symposium, Noordwijk, The Netherlands, 25-27 September 2013.

(C) 2020 by the authors. Licensee MDPI, Basel, Switzerland. This article is an open access article distributed under the terms and conditions of the Creative Commons Attribution (CC BY) license (http://creativecommons.org/licenses/by/4.0/). 